\title{
Transplantation of Neural Progenitor Cells within Hyaluronic Acid Hydrogel in Traumatic Brain Iniury in Experiment
}

DOI: $10,17691 / \mathrm{stm} 20179.4 .13$

ReceivedMay 12, 2017

A.V. Balyabin, MD, PhD, Researcher, Neurosurgery Department';

O.P. Tikhobrazova, PhD, Senior Researcher, Experimental Modeling Department, Central Research Laboratory²;

A.A. Gladkov, Junior Researcher, Laboratory of Neuroengineering, Center for Translational Technologies;; Junior Researcher, Molecular and Cellular Technologies Department, Central Research Laboratory2;

M.S. Muravyova, PhD Student ${ }^{3}$;

Y.A. Klyuyey, Intern, Department of Radiodiagnostics, Faculty of Advanced Medical Training2;

1.V. Mukhina, DSc, Professor, Head of the Central Research Laboratory²; Head of the Department of Normal

Physiology named after N.Y Belenkov2; Professor, Neurotechnology Department, Institute of Biology

and Biomedicine ${ }^{3}$; Head of the Center for Translational Technologies ${ }^{3}$; Leading Researcher of Biotechnologies Group ${ }^{1}$

${ }^{1}$ Privolzhsky Federal Research Medical Center, Ministry of Health of the Russian Federation,

18 Verkhne-Volzhskaya naberezhnaya, Nizhny Novgorod, 603155, Russian Federation;

${ }^{2}$ Nizhny Novgorod State Medical Academy, 10/1 Minin and Pozharsky Square, Nizhny Novgorod, 603005,

Russian Federation;

${ }^{3}$ Lobachevsky State University of Nizhni Novgorod, 23 Prospekt Gagarina, Nizhny Novgorod, 603950,

Russian Federation

The aim of the study was to evaluate the possibilities of transplantation of autologous neural progenitor cells from C57BL/6 mouse nasal olfactory lamina propria in the hydrogel based on low-, medium-, and high-molecular weight hyaluronic acid during a reconstructive operation after an open traumatic brain injury (TBI) in experiment.

Materials and Methods. A reconstructive therapy was performed on the model of an open traumatic brain injury using the autologous neural progenitor cells from C57BL/6 mouse nasal olfactory lamina propria in a hydrogel based on high, medium, and low molecular hyaluronic acid 7 days after injury. Short-term and long-term impairments of neurological functions and memory due to TBI were assessed using a series of behavioral and cognitive tests (modified neurological severity score (mNSS)), open field test, novel object recognition test and passive avoidance behavior). High-field magnetic resonance tomography was used for visualization of a lesion cavity.

Results. Implantation of autologous neural progenitor cells in the hydrogel based on high-molecular weight hyaluronic acid in contrast to low- and medium-molecular weight had a protective effect reducing neurological deficit and restoring motor functions, short-term and long-term memory in the posttraumatic period. The effect of hydrogel molecular weight on the morphological characteristics of the injury cavity was less effective during the autologous neural progenitor cells transplantation after TBI.

Conclusion. Transplantation of autologous neural progenitor cells from C57BL/6 mouse nasal olfactory lamina propria in the hydrogel based on high-molecular weight hyaluronic acid into the injury focus after open brain trauma promotes functional recovery of reflex and cognitive behavior of animals in the posttraumatic period.

Key words: autologous neural progenitor cells; hyaluronic acid hydrogel; traumatic brain injury; TBI; functional recovery.

Treatment of the consequences of traumatic brain injury (TBI) and a number of other neurodegenerative processes is one of the most complex and socially significant problems of medicine, as in the majority of cases it leads to disability and decline in cognitive capacity [1, 2]. Absence of significant success of drug therapy [3], limited ability of neurogenesis in adults organism [4] cause the study of fundamental regeneration mechanisms of the injured brain areas and developing principally new methods of TBI treatment. The development of adequate matrix-carrier for transplanted cells, which would create a specific microenvironment with prolonged recovery process of neural networks and tissue structure regeneration is one of the most promising methods of the treatment of TBI [5-7].

At present, the works in the field of neurotransplantation are mainly focused on the use of hydrogel systems of various composition with progenitor cells. Firstly, the pore structure of the hydrogel allows cells and nutrients to penetrate into the matrix and metabolic products to dispose in the volume of the body [8]. Second, the hydrogel systems are optimal in terms of

For contacts: Olga P. Tikhobrazova, e-mail: olga.tikhobrazova@gmail.com 
mechanical compatibility with the tissues of the brain and spinal cord tissue. The compatibility of the mechanical characteristics is determining during the differentiation of progenitor cells [9] and influences overall success of implantation [10]. Third, hydrogels are promising drug compounds in the injury site.

Extracellular matrix of the brain consists of the molecules synthesized and secreted by neurons and glial cells, which form stable aggregates in intercellular space in various combinations [11]. The most pronounced and studied clusters of extracellular matrix molecules in the CNS are perineuronal nets [12]. They are rich in hyaluronic acid $(\mathrm{HA})$, chondroitin sulfate proteoglycans and link proteins. HA being a framework for the extracellular matrix take part in regulation of cellular differentiation, migration, proliferation, and angiogenesis. Perisynaptic matrix comprising HA can create barriers to the diffusion of synaptic molecules in the membrane and thus promoting compartmentalization synaptic mechanism of signal transmission.

In addition, HA can form hydrogels, which not only contain a large amount of water that promotes the viability of cells, but also have biophysical, biochemical properties, similar to those of the body tissues. Moreover, hyaluronic hydrogels are biologically compatible. However, their use for neurotransplantation is not yet sufficiently developed.

The aim of the investigation was to assess the possibilities of transplantation of autologous neural progenitor cells of C57BL/6 mouse nasal olfactory lamina propria in the hydrogel based on low-, medium-, and high-molecular weight hyaluronic acid hydrogel in the reconstructive surgery of open traumatic brain injury in experiment.

Materials and Methods. Adult male C57BL/6 mice (10-12 weeks of age, 20-22 g) were purchased from the Department of Experimental Animals, Pushchino, Russia, and maintained under standard housing conditions (temperature: $18-24^{\circ} \mathrm{C}$; relative humidity: $45 \%$; light and dark cycle: 12:12 h). Food and water were provided ad libitum. All animals were treated according to the protocols evaluated and approved by the Guide for the Care and Use of Laboratory Animals (National Research Council, 2011), Russian Federation standard 33044-2014 "Principles of Good Laboratory Practice" and the Ethical Committee of the Nizhny Novgorod State Medical Academy. The number of mice used and their suffering were minimized.

Hyaluronic acid-based hydrogel. The hydrogel which was used for neurotransplantation was a sterile $1 \%$ water solution of sodium hyaluronate (Scientific Production Enterprise (SPE) Tula Industry, Ltd, Russia) with different molecular weight of the active substance: low-molecular weight form $(55.0 \mathrm{kDa})$; mediummolecular weight form (500.0 kDa); high-molecular weight form $(1,250.0 \mathrm{kDa})$. Sodium chloride $(8.5 \mathrm{mg})$ and phosphate buffered saline (PBS) $(320 \mu \mathrm{g} / \mathrm{ml})$ were used as auxiliary substances.
Culture preparation. Neural progenitor cells were obtained from the olfactory mucosa of C57BL/6 mice according to the previously developed protocol [13].

Weight-drop traumatic brain injury model. In this study, we used the Feeney weight-drop method with some modifications [14] by dropping the weight with a blunt surface which provides acceleration with a minimum of local action at the point of application of the traumatic force. This model produces a complete clinical picture of focal injury including brain contusion, and allows exploration of traumatic and local changes that are accompanied by secondary death of neurons in distant parts of the brain, which are sensitive to trauma, such as hippocampus, dentate gyrus and thalamus, and also evaluation of the movement disorders and cognitive impairment.

Spontaneously breathing mice were anesthetized with $1.5 \%$ isoflurane in oxygen and placed in a stereotaxic frame adapted for mice (Narishige, Japan) on a $37.8^{\circ} \mathrm{C}$ heating pad. For inducing focal brain injury, the animals were placed on a non-flexible steel platform in order to minimize energy dissipation, to reduce the risk of jaw fractures and achieve the horizontal location of the calvarium to the tip of the weight. All animals underwent craniotomy under aseptic conditions using a surgical microscope. The scalp was shaved and cleansed with chlorhexidine and povidone-iodine wash. The scalp and periosteum were opened, and left craniotomy $(4 \mathrm{~mm}$ in diameter, $2 \mathrm{~mm}$ posterior to stereotaxic bregma, and $2 \mathrm{~mm}$ lateral to the midline) was performed with a fine drill, leaving the dura intact. A weight-drop device was placed over the dura. A sterile polypropylene cylindrical tube was manufactured with a 4-mm opening at one end. Then, a $4 \mathrm{~g}$ weight stainless steel cylinder was dropped from $80 \mathrm{~cm}$ height through a guiding tube onto the burr hole to create a contusion brain injury model. $\mathrm{TBI}$ results in an ipsilateral injury with cortical contusion, hemorrhage, dura rupture that is typical for severe head trauma. After injury, the scalp incision was closed tightly with polypropylene suture and treated with an antiseptic solution.

Experimental groups. Adult male C57BL/6 mice $(n=60)$ were randomly assigned to six groups. The first group $(n=10)$ included intact animals. The second group $(n=10)$ comprised sham-operated animals. Mice of control third group $(n=10)$ were injected PBS into the lesion cavity. The fourth group $(n=10)$ was with implantation of progenitor cells in the high-molecular weight hyaluronic acid hydrogel (HA "D"); the fifth group - implantation of progenitor cells in the mediummolecular weight hyaluronic acid hydrogel (HA "E") $(n=10)$; the sixth group - implantation of progenitor cells in the low-molecular weight hyaluronic acid hydrogel (HA "K") (n=10).

Injury day was considered as day 0 . Mice in the "HA hydrogel with cells" groups were subjected to TBI, and 7 days later, a hyaluronic acid-based hydrogel with engrafted progenitor cells $(30 \mu \mathrm{l})$ was directly 
transplanted into the injury core. Mice of the control group underwent $\mathrm{TBI}$ with the injections of the PBS vehicle $(30 \mu \mathrm{l})$ only. The animals of intact group were not subjected to anesthesia, modeling $\mathrm{TBI}$ and transplantation. Sham-injured mice received similar surgical procedures including a craniotomy without TBI.

Morphofunctional parameters of the assessment of cell transplantation in the studied carriers in vivo were based on magnetic resonance imaging (MRI) analysis and testing of neurological and cognitive functions of mice.

The modified neurological severity score. This scale includes the assessment of 10 clinical parameters characterizing motor functions, ability to balance, and some behavioral reactions of animals (alertness, searching behavior). One point is given for failure of the task and no point for succeeding. A maximal neurologic severity score of 10 points indicates severe neurological dysfunction, with failure at all tasks [15-18].

A day before TBI modeling, neurological status was evaluated in all groups of animals to assess the initial level and then on 1-10, 15, 20, 30 days post injury.

Open field test. To assess cortical function, mice were subjected to behavioral testing using of the open field task on day 4 prior to injury (baseline) and on days 10 and 30 post injury. Locomotor and exploratory behavior was analyzed. At the beginning of the test, individual animals were placed in the center of the open field box (LE800S; PanLab/Harvard Apparatus Spain; Stoelting Co., USA) and allowed to freely explore it for 5 min. Traveled distance, mean speed and the number of rearing as well as duration of anxiety-like behavior such as grooming and freezing acts were automatically recorded with a video camera (SONY SSC-G118; Japan). The data acquisition and analysis were controlled with the SMART v.3.0.03 software (Panlab/ Harvard Apparatus Spain; Stoelting Co., USA).

Passive avoidance test. The passive avoidance task was used to assess simple non-spatial learning ability on day 10 post injury [19]. The passive avoidance device comprised of two adjoining compartments, one illuminated and one darkened, divided by a guillotine door. The dimensions of each compartment were $46 \times 27 \times 25 \mathrm{~cm}$. The floor of the compartments consisted of steel rods capable of delivering a slight electric foot shock (50 Hz, $1.5 \mathrm{~mA}$ for $10 \mathrm{~ms}$ ) to the animal (Shuttle Box LE895; PanLab/Harvard Apparatus Spain; Stoelting Co., USA). The test began by placing the mouse in the lighted compartment. The feeding of electric foot shock, the position of the guillotine door between the compartments and the latency for crossing to the dark compartment were recorded with the Shutavoid v.1.8.03 software (PanLab/Harvard Apparatus Spain; Stoelting Co., USA). The latency was recorded with the open door between the compartments. The test was over when the animal entered the dark compartment or did not do it during $3 \mathrm{~min}$. Cognitive functions were studied during retention test after $24 \mathrm{~h}$ (on day 10) and on day 30 of postraumatic period.
Novel object recognition test. To evaluate impairments of nonspatial hippocampus-mediated short-term memory, a novel object recognition test was performed. The procedure consisted of three sessions - habituation, training, and retention [20, 21]. On days 23 and 24 after TBI, the animals were placed in the center of the square arena $(45 \times 45 \times 40 \mathrm{~cm})($ LE802S; PanLab/Harvard Apparatus, Spain; Stoelting Co., USA) for $10 \mathrm{~min}$ for habituation and stress reduction - the animals explored the empty arena. On day 24 of the posttraumatic period, in the first trial, two identical objects were placed in the right and left corners of the box. Mice were placed into the box for $10 \mathrm{~min}$, where they explored the two objects, and the exploratory activity was recorded. During the retention session $24 \mathrm{~h}$ after the training session, the animals were placed into the same box, but one of the familiar objects used during the training was replaced with a novel object. The objects were different color but similar in size. The animals were then allowed to explore freely for $10 \mathrm{~min}$, and the time spent in exploring each object was recorded.

In the course of the experiment, the cumulative time spent by the mouse exploring each of the familiar and novel objects was recorded. Exploration of an object was defined as follows: directing the nose to the object at a distance of $2 \mathrm{~cm}$ and touching it with the nose. Animal behavior during training, and retention was considered as explorative if total exploring time to the objects lasted not less than $10 \mathrm{~s}$. Registration of single explorative acts of animals was carried out using video camera (SONY SSC-G118; Japan). The data acquisition and analysis were controlled with the SMART v.3.0.03 software (Panlab/Harvard Apparatus Spain; Stoelting Co., USA). Memory function was operationally defined by the discrimination ratio (DIR) for the novel object (discrimination ratio, DIR) by the formula: DIR=[(novel object exploration time - familiar object exploration time)/total exploration time] $\times 100$ [17].

Magnetic resonance imaging. Imaging of the mice brain was carried out on the high field MRI machine Agilent Technologies DD2-400 9.4 T (USA) $\left(400 \mathrm{MHz}\right.$ ) with a volume coil M2M $\left(\mathrm{H}_{1}\right)$. Monitoring of the physiological parameters of animals (temperature, respiration and ECG) during imaging was performed on a SA Instruments company (USA) equipment using PCSAM program. During imaging the animals were under isoflurane anesthesia. Animals were heated by $37^{\circ} \mathrm{C}$ warm air.

MGEMS (multi gradient echo multi slice) pulse sequence with the following parameters: repetition time is $1,300 \mathrm{~ms}$, echo time is $8 \mathrm{~ms}$, echo number 8 , the amount of savings -4 , number of slices -15 , the thickness of slice was $1 \mathrm{~mm}$, the field of vision $20 \times 20 \mathrm{~mm}^{2}$, matrix size $-128 \times 128$ pixels was used for $\mathrm{T}^{*}$-weighted imaging. The total duration of the sequence was 8 min $32 \mathrm{~s}$.

Statistical analysis. Results were expressed as mean \pm standard error of the mean (SEM). All data 
were evaluated for normal distribution by Shapiro-Wilk normality test (W-criterion). All data were analyzed using Statistica 8.0 software (StatSoft, USA). Nonparametric analyses were performed using a Wilcoxon signedrank test for repeated measures of outcomes of the TBI. Statistical comparisons between behavioral data of treatments on each evaluated time-point were performed using a Mann-Whitney $U$ test. A p value of $<0.05$ was considered significant.

\section{Results and Discussion}

Assessment of neurological status of the animals in the posttraumatic period. The functional assessment for mice behavior showed the sustained fault of neurological functions on day 1 after TBI (Figure 1). The modified neurological severity score in the groups with brain injury was significantly higher compared with initial level as well as intact and shamoperated groups of animals $(p<0.05)$.

Asymmetrical behavior was characteristic of all animals due to limb paresis on the contralateral side to the injury cavity, impairments of motor functions, inability to perform reactions connected with maintaining the balance and movement coordination, which is the evidence of the damage of the motor cortex, midbrain, and pons Varolii. Impairments of reflexes associated with the midbrain and cortex damage were also observed.

The neurological deficit was reduced by day 10 of the posttraumatic period in all groups of animals with transplantation of autologous progenitor cells in $\mathrm{HA}$ hydrogel into the injury cavity on day 7 after TBI. However, no statistically significant differences compared to the control group were detected $(p>0.05)$.

The tendency to the decrease of the neurological deficit in the group with implantation of the HA "D" hydrogel with engrafted neural progenitor cells into the injury cavity was observed on day 20 of the posttraumatic period $(p>0.05)$.

The neurological status of mice in this group significantly improved compared to the control group (PBS) $(p<0.05)$ in contrast from the groups with implantation into the lesion site on the day 7 of the posttraumatic period of neural cells in the HA "E" and HA "K" on the day 30 after injury. It should be noted that throughout the observed posttraumatic period the neurological functions in animals with TBI were not completely restored. Paresis on the contralateral side to injury and partial impairments of reflexes (searching behavior) were observed The level of neurological deficit of mice was significantly increased compared to the intact and shamoperated groups of animals.

Open field test. A preliminary testing of the animals (before TBI) did not reveal any differences between the groups in the main behavior characteristics. TBI resulted in the impairment of the animal behavior structure. The data obtained in the open field test showed statistically significant the motor and explorative activity decreasing in comparison with the initial level also as the values in the intact and sham-operated groups of the animals $(p<0.05)$. There was no restoration of exploratory activity in any of the mice groups with TBI.

The activity speed recovery occurred in the group of animals with implantation of neural progenitor cells based on HA "D" hydrogel into the injury cavity on day 10 of the posttraumatic period. This factor was significantly higher compared to the control group $(p<0.05)$, but did not differ either from the values of the intact and shamoperated group or from other experimental groups with TBI $(p>0.05)$ (Figure 2).

On day 30 of the posttraumatic period, the speed of motor activity in this group was statistically higher compared to the control (PBS) mice group $(p<0.05)$. It signified the restoration of locomotor activity and functions associated with maintaining the balance.

The activity speed was not restored in the groups of mice with implantation of HA "E" and HA "K" hydrogels engrafted with neural progenitor cells and was not different from the value of the control (PBS) group

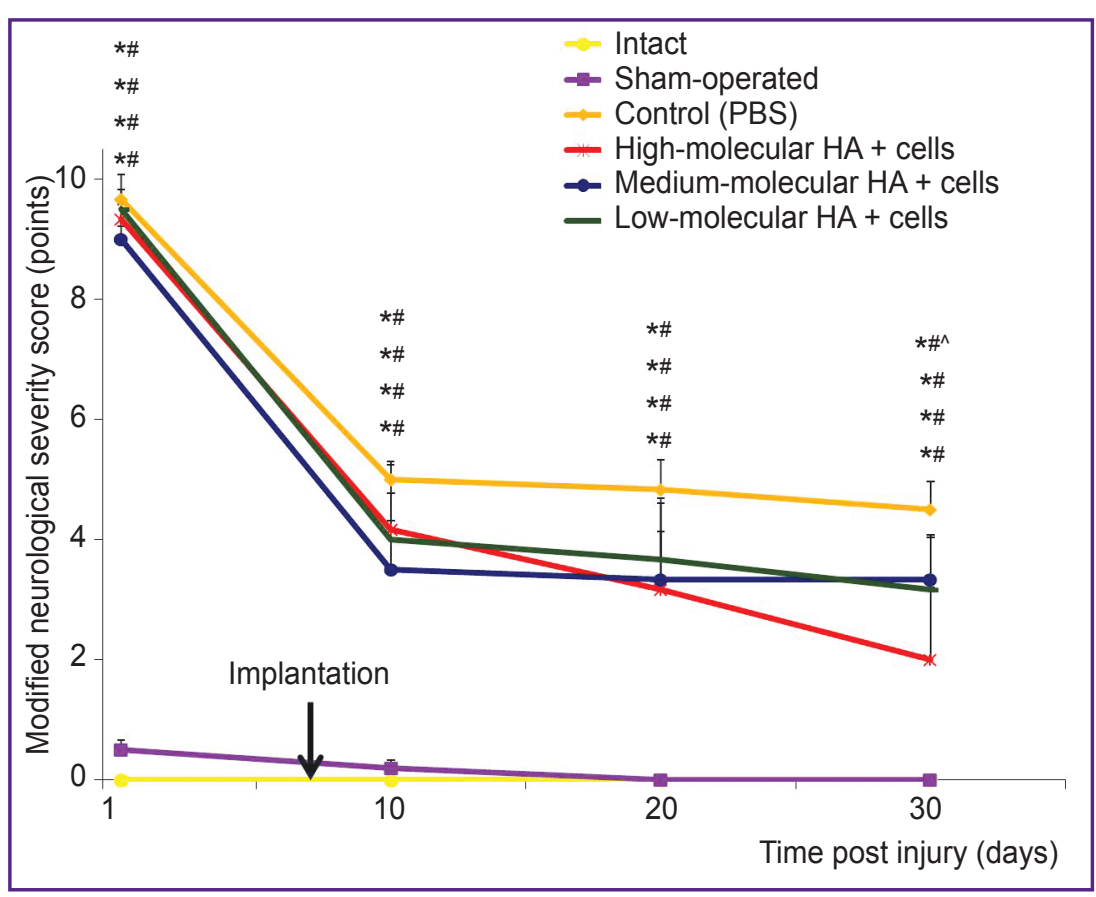

Figure 1. The neurological status (modified neurological severity score) of C57BL/6 mice in the posttraumatic period

* $p<0.05$ significant difference compared with the intact group, Mann-Whitney $U$ test; ${ }^{\#} p<0.05$ significant difference compared with the sham-operated group; Mann-Whitney $U$ test; ${ }^{\wedge} p<0.05$ significant difference compared with the control (PBS) group; Mann-Whitney $U$ test; $n=10$ per group 

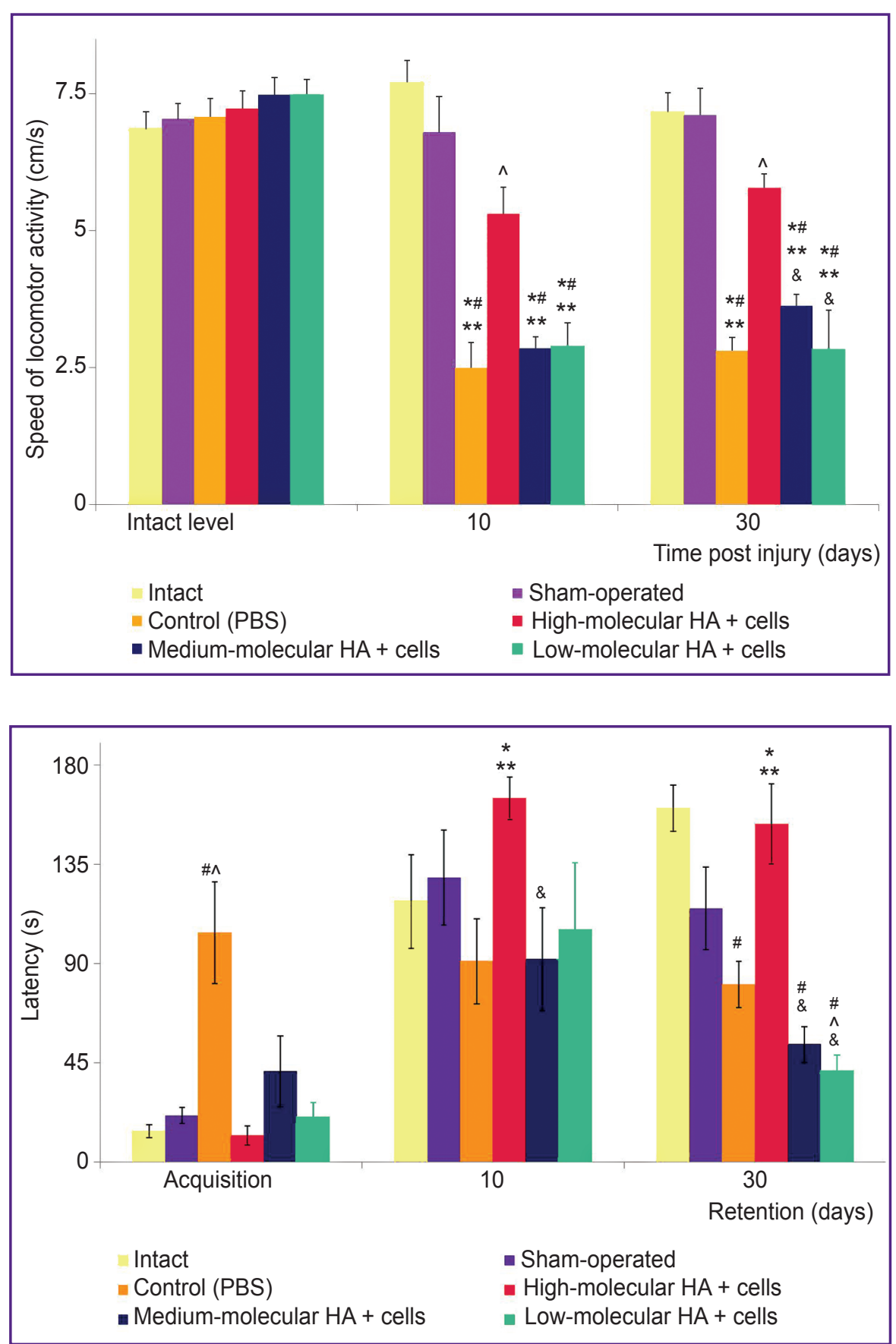

Figure 3. The effect of hyaluronic acid hydrogel engrafted with neuron progenitor cells implantation on passive avoidance behavior of C57BL/6 mice in the posttraumatic period

${ }^{*} p<0.05$ values of the time spent at light compartment during retention test significantly differ compared from values during acquisition session, Wilcoxon test; \# $p<0.05$ significant difference compared to intact mice, Mann-Whitney $U$ test; ^ $p<0.05$ significant difference compared to sham-operated group; Mann-Whitney $U$ test; ${ }^{* *} p<0.05$ significant difference compared with the control (PBS) group; Mann-Whitney $U$ test; \& $p<0.05$ significant difference compared with the group of high-molecular weight $\mathrm{HA}+$ cells, Mann-Whitney $U$ test; $n=10$ per group

( $p>0.05)$. Moreover, the activity speed was significantly lower in compared with the group with implantation of the neural cells in the HA "D" hydrogel $(p<0.05)$.
Figure 2. The speed of locomotor activity of C57BL/6 mice in the open field test in the posttraumatic period

* $p<0.05$ significant differences between pre- and post-injured values, Wilcoxon test; \# $p<0.05$ values in injured brain mice differ significantly from the values of the intact mice, Mann-Whitney $U$ test; ** $p<0.05$ significant difference compared to sham-operated group, Mann-Whitney $U$ test; ${ }^{\wedge} p<0.05$ significant difference compared with the control (PBS) group; Mann-Whitney U test; \& $p<0.05$ significant difference compared with the group of high-molecular weight $\mathrm{HA}+$ cells, Mann-Whitney $U$ test; $\mathrm{n}=10$ per group

Passive avoidance behavior. The results of behavioral tests revealed the impairment of learning processes of animals in the control (PBS) group as a result of TBI. The entry latency to the dark compartment in the control (PBS) group of mice significantly increased compared to intact and sham-operated group $(p<0.05)$ during the acquisition test. It was caused by the impairment of the motor functions of the CNS of animals owing to the TBI.

On day 10, the entry latency into the dark compartment of the shuttle box in the group with neural progenitor cells in HA "D" hydrogel into the injury cavity significantly increased in the retention test compared with acquisition session as well as in intact and sham-operated groups $(p<0.05)$. Moreover, the latency in this group was statistically higher compared to the control (PBS) group $(p<0.05)$ (Figure 3$)$.

In the groups with the implantation hydrogel of mediumand low-molecular weight $\mathrm{HA}$ engrafted with the progenitor cells into injury cavity 1 week after TBI, the time spent in the illuminated part of chamber at the retention test compared with acquisition session did not differ 
$(p>0.05)$. At the same time, the latency in the group with the implantation of cells in the hydrogel of low-molecular weight HA was significantly reduced compared to the group of high-molecular weight HA hydrogel engrafted with the cells $(p<0.05)$.

Long-term memory functions were assessed by ability of mice to retain passive avoidance behavior on day 30 of the posttraumatic period. The entry latency at the retention test significantly differed from acquisition session $(p<0.05)$ in the group of animals with implantation of progenitor cells based on HA "D" hydrogel into the injury cavity. Moreover it was significantly exceeded the time of the control (PBS) group $(p<0.05)$

In the groups with transplantation of neural cells in the medium- and low-molecular weight HA hydrogel as well as in the control group the time spent in the lite compartment was significantly less than the time in the intact group $(p<0.05)$ and in the group with implantation of progenitor cells in the hydrogel of the high-molecular weight HA $(p<0.05)$ on day 30 of the posttraumatic period.

Thus, implantation of HA "D" hydrogel engrafted with progenitor cells into the injury cavity 7 days after TBI modeling caused an optimizing effect on the ability of the animals to acquire conditioned reflexes on day 10 and to update traces of long-term memory in the remote period.

Novel object recognition test. The data obtained showed that $\mathrm{TBI}$ results in the significant impairment of hippocampus-dependent working memory. Discrimination ratio in the control (PBS) group was significantly decreased compared to the intact and the sham-operated groups $(p<0.05)$ (Figure 4).

The time spent to explore the novel object was statistically higher than the time spent for the familiar object in the group of mice with progenitor cells in HA "D" hydrogel implantation on day 7 following the injury. Discrimination ratio in this animal group did not differ from that in the intact and sham-operated groups of mice over the whole posttraumatic period. At the same time, the DIR value in the group with progenitor cells in HA "D" hydrogel implantation was increased compared to control (PBS) group $(p<0.05)$ that indicates the recovery of CA1-CA3 field hippocampus-dependent short-term recognition memory functions.

In the groups of mice with the neural cells in the hydrogel of medium- and low-molecular weight HA implantation after 7 days after TBI, the discrimination ratio, on the contrary, was not differ from the control (PBS) group $(p>0.05)$. It was significantly decreased in compare to the value in the group of high-molecular weight HA hydrogel engrafted with progenitor cells implantation as well as in the intact and sham-operated groups $(p<0.05)$.

Magnetic resonance imaging data. MRI data obtained 30 days after injury showed that in all groups with implantation of neural progenitor cells with HA hydrogel into the injury cavity the volume of the lesion

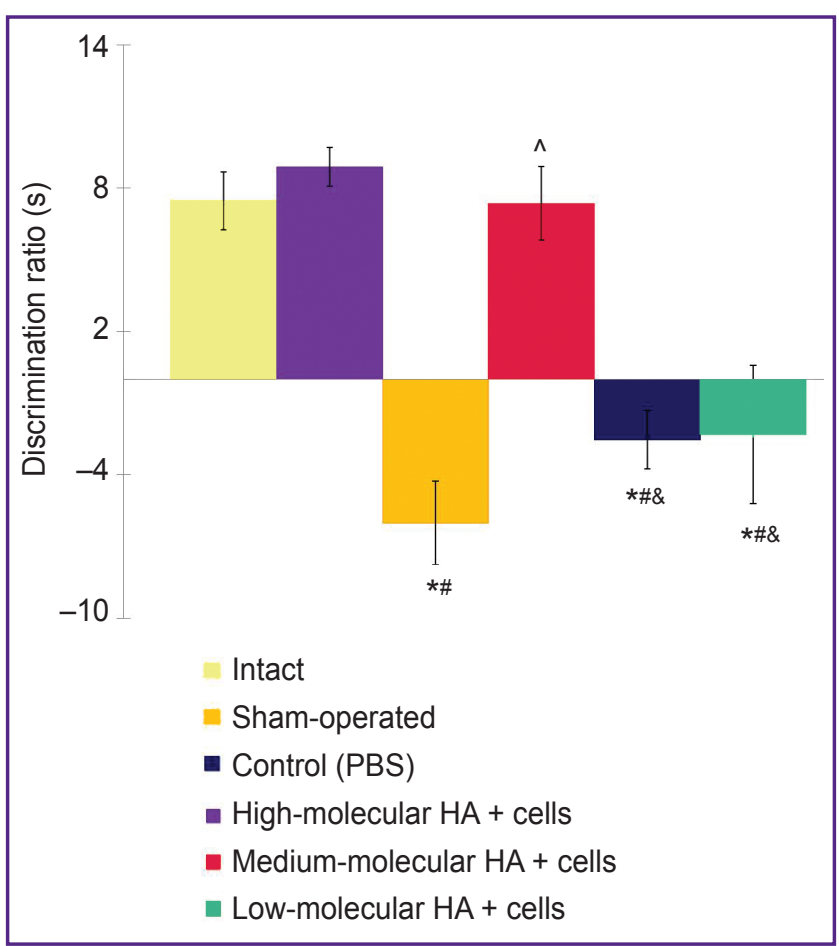

Figure 4. The recovery of C57BL/6 mice recognition memory in the posttraumatic period

Statistical analysis of the discrimination ratio values of the novel object recognition test results revealed a significant difference between the groups: * $p<0.05$ compared to intact group, Mann-Whitney $U$ test; \# $p<0.05$ compared to shamoperated group, Mann-Whitney $U$ test; ${ }^{\wedge} p<0.05$ compared to control (PBS) group, Mann-Whitney U test; \& $p<0.05$ compared with the group of high-molecular weight HA + cells, MannWhitney $U$ test; $n=10$ per group

area did not significantly differ from the control (PBS) group $(p<0.05)$ (Figure 5).

The injury area was detected as a defect on the surface of the hemisphere on the magnetic resonance images on day 30 of the posttraumatic period in the group with implantation of the progenitor cells in the HA hydrogel " $D$ ", while the lesion area had a rounded form in the sensorimotor cortex in the group with progenitor cells transplanted with the HA hydrogel "E" . Cystic lesion of a round form with irregular margins filled with fluid in the area of the sensorimotor cortex was visualized on the magnetic resonance images of the group of mice with implantation of HA "K" hydrogel engrafted with progenitor cells.

Thus, TBI results in neurologic, motor and cognitive behavior of animals impairments. Implantation of neural progenitor cells in the hydrogel of the high-molecular weight HA into the injury cavity 7 days after TBI had the protective effect by restoring the synaptic plasticity (cortex, hippocampus) underlying the motor functions, learning and memory processes, in contrast to the transplantation of the medium- and low-molecular weight HA hydrogel with engrafted neural progenitor cells. 


\section{EXPERIMENTAL INVESTIGATIONS}

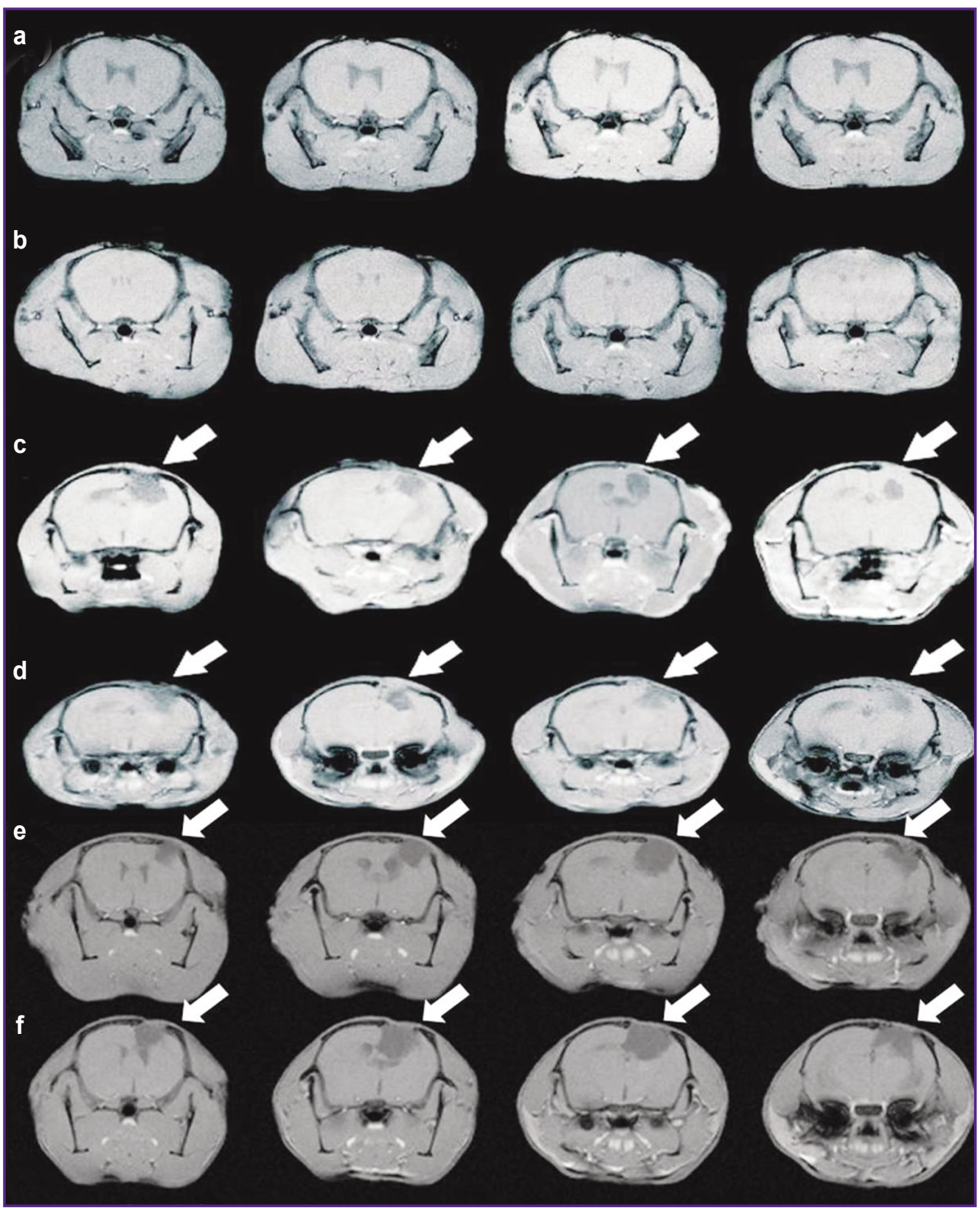

Figure 5. Magnetic resonance images of the brain slices on day $\mathbf{3 0}$ of the posttraumatic period: (a) intact mice brain; (b) sham-operated mice brain; (c) control mice brain (PBS) with traumatic brain injury. Mice with traumatic brain injury and implanted in injury area: (d) cells in the high-molecular weight hyaluronic hydrogel; (e) cells in the medium-molecular weight hyaluronic hydrogel; (f) cells in the low-molecular weight hyaluronic hydrogel. T2*-weighted images were obtained using $9.4 \mathrm{~T}$ MRI machine, pulse sequence MGEMS. The trauma area is indicated by the arrows

A facilitative effect of transplantation of neural progenitor cells in HA hydrogel on the ability of the animals to acquire passive avoidance reflex in acute posttraumatic period and to update traces of short and long-term memory in the remote period has been established. The effect of implantation of the autologous neural progenitor cells in HA hydrogel on the restoration of morphological parameters of the injured brain tissue was less evident.
However, an attempt to regenerate the brain tissue structure was observed in the group with implantation of progenitor cells in the hydrogel of the high-molecular weight $\mathrm{HA}$.

Conclusion. Transplantation of autologous neural progenitor cells from C57BL/6 mouse nasal olfactory lamina propria in the hydrogel based on high-molecular weight hyaluronic acid into the injury cavity after open 
brain trauma promotes functional recovery of reflex and cognitive behavior of animals in the posttraumatic period.

Study Funding. The research was supported by the Federal Target Program "Research and development in priority areas of the development of the scientific and technological complex of Russia for 2014-2020" of the Ministry of Education and Science of Russia (Project ID RFMEFI60715X0117).

Conflicts of Interest. The authors have no conflicts of interest.

\section{References}

1. Gusev E.I., Konovalov A.N., Skvortsova V.I., Gekht A.B. Nevrologiya [Neurology]. Moscow: GEOTAR-Media; 2009; $1040 \mathrm{p}$.

2. Bullock M.R., Chesnut R., Ghajar J., Gordon D., Hartl R., Newell D.W., Servadei F., Walters B.C., Wilberger J.E. Guidelines for the surgical management of traumatic brain injury author group. Neurosurgery 2006; 58(3): S2-Vi, https:// doi.org/10.1093/neurosurgery/58.3.vi.

3. Mayo Clinic Staff. Treatments and drugs. In: Traumatic brain injury. Mayo Clinic; 2014. URL: https://www.mayoclinic.org/ diseases-conditions/traumatic-brain-injury/basics/treatment/con20029302.

4. Dobrowolski S., Lepski G. Stem cells in traumatic brain injury. Am J Neurosci 2013; 4(1): 13-24, https://doi. org/10.3844/amjnsp.2013.13.24.

5. Crompton K.E., Goud J.D., Bellamkonda R.V., Gengenbach T.R., Finkelstein D.I., Horne M.K., Forsythe J.S. Polylysine-functionalised thermoresponsive chitosan hydrogel for neural tissue engineering. Biomaterials 2007; 28(3): 441449, https://doi.org/10.1016/j.biomaterials.2006.08.044.

6. Tate C.C., Shear D.A., Tate M.C., Archer D.R., Stein D.G., LaPlaca M.C. Laminin and fibronectin scaffolds enhance neural stem cell transplantation into the injured brain. J Tissue Eng Regen Med 2009; 3(3): 208-217, https://doi. org/10.1002/term.154.

7. Mo L., Yang Z., Zhang A., Li X. The repair of the injured adult rat hippocampus with NT-3-chitosan carriers. Biomaterials 2010; 31(8): 2184-2192, https://doi.org/10.1016/j. biomaterials.2009.11.078.

8. Cao Z. Developing chitosan-based biomaterials for brain repair and neuroprosthetics. Master's Thesis. University of Tennessee; 2010. URL: http://trace.tennessee.edu/utk gradthes/609/.

9. Engler A.J., Sen S., Sweeney H.L., Discher D.E. Matrix elasticity directs stem cell lineage specification. Cell 2006; 126(4): 677-689, https://doi.org/10.1016/j.cell.2006.06.044.

10. Liao H., Munoz-Pinto D., Qu X., Hou Y., Grunlan M.A., Hahn M.S. Influence of hydrogel mechanical properties and mesh size on vocal fold fibroblast extracellular matrix production and phenotype. Acta Biomater 2008; 4(5): 11611171, https://doi.org/10.1016/j.actbio.2008.04.013.

11. Wlodarczyk J., Mukhina I., Kaczmarek L., Dityatev A.
Extracellular matrix molecules, their receptors, and secreted proteases in synaptic plasticity. Dev Neurobiol 2011; 71(11): 1040-1053, https://doi.org/10.1002/dneu.20958.

12. Vedunova M., Sakharnova T., Mitroshina E., Perminova M., Pimashkin A., Zakharov Y., Dityatev A., Mukhina I. Seizure-like activity in hyaluronidase-treated dissociated hippocampal cultures. Front Cell Neurosci 2013; 7: 149, https://doi.org/10.3389/fncel.2013.00149.

13. Balyabin A.V., Tikhobrazova O.P., Muravyeva M.S., Klyuev E.A., Ponyatovskaya A.V., Shirokova O.M., Bardakova K.N., Minaev N.V., Koroleva A.V., Mitaeva Y.I., Mitroshina E.V., Vedunova M.V., Rochev Y.A., Chichkov B.N., Timashev P.S., Bagratashvili V.N., Mukhina I.V. Long-term neurological and behavioral results of biodegradable scaffold implantation in mice brain. Sovremennye tehnologii $v$ medicine 2016; 8(4): 198-211, https://doi.org/10.17691/stm2016.8.4.25.

14. Feeney D.M., Boyeson M.G., Linn R.T., Murray H.M., Dail W.G. Responses to cortical injury: I. Methodology and local effects of contusions in the rat. Brain Res 1981; 211(1): 67-77, https://doi.org/10.1016/0006-8993(81)90067-6.

15. Beni-Adani L., Gozes I., Cohen Y., Assaf Y., Steingart R.A., Brenneman D.E., Eizenberg O., Trembolver V., Shohami E. A peptide derived from activity-dependent neuroprotective protein (ADNP) ameliorates injury response in closed head injury in mice. J Pharmacol Exp Ther 2001; 296(1): 57-63.

16. Levy A., Bercovich-Kinori A., Alexandrovich A.G., Tsenter J., Trembovler V., Lund F.E., Shohami E., Stein R., Mayo L. CD38 facilitates recovery from traumatic brain injury. J Neurotrauma 2009; 26(9): 1521-1533, https://doi. org/10.1089/neu.2008.0746.

17. Beni-Adani L., Eizenberg O., Cohen Y. Correlation between neurological severity score and T2-weighted MRI in head injured mice. Rest Neurol Neurosci 2000; 16(3-4): 242.

18. Tsenter J., Beni-Adani L., Assaf Y., Alexandrovich A.G., Trembovler V., Shohami E. Dynamic changes in the recovery after traumatic brain injury in mice: effect of injury severity on T2-weighted MRI abnormalities, and motor and cognitive functions. J Neurotrauma 2008; 25(4): 324-333, https://doi. org/10.1089/neu.2007.0452.

19. Bures J., Bureshova O., Huston J.P. Metodiki i osnovnye eksperimenty po izucheniyu mozga $i$ povedeniya [Techniques and basic experiments on the study of the brain and behavior]. Moscow: Vysshaya shkola; 1991; 399 p.

20. Huang T.N., Chuang H.C., Chou W.H., Chen C.Y. Wang H.F., Chou S.J., Hsueh Y.P. Tbr1 haploinsufficiency impairs amygdalar axonal projections and results in cognitive abnormality. Nat Neurosci 2014; 17(2). 240-247, https. Ildoi. org/10.1038/nn.3626.

21. Moscardo E., Salvetti B., Becchi S., Bertini G., Fabene P.F. The novel object recognition test in rodents: which are the essential methodological aspects? In: Proceedings of Measuring Behavior 2012, 8 th International Conference on Methods and Techniques in Behavioral Research. Spink A.J., Grieco F., Krips O.E., Loijens L.W.S., Noldus L.P.J.J., Zimmerman P.H. (editors). The Netherlands; 2012, p. 476-478. 